\title{
Zagreb Connection Indices of Molecular Graphs Based on Operations
}

\author{
Jinde Cao $\mathbb{D},{ }^{1}$ Usman Ali, ${ }^{2}$ Muhammad Javaid $\left(\mathbb{D},{ }^{2}\right.$ and Chuangxia Huang $\mathbb{D}^{3}$ \\ ${ }^{1}$ School of Mathematics, Southeast University, Nanjing 210096, China \\ ${ }^{2}$ Department of Mathematics, School of Science, University of Management and Technology, Lahore, Pakistan \\ ${ }^{3}$ School of Mathematics, Changsha University of Science and Technology, Changsha 410114, China
}

Correspondence should be addressed to Jinde Cao; jdcao@seu.edu.cn

Received 15 November 2019; Accepted 5 February 2020; Published 30 March 2020

Academic Editor: Qingling Wang

Copyright (C) 2020 Jinde Cao et al. This is an open access article distributed under the Creative Commons Attribution License, which permits unrestricted use, distribution, and reproduction in any medium, provided the original work is properly cited.

Topological index (numeric number) is a mathematical coding of the molecular graphs that predicts the physicochemical, biological, toxicological, and structural properties of the chemical compounds that are directly associated with the molecular graphs. The Zagreb connection indices are one of the TIs of the molecular graphs depending upon the connection number (degree of vertices at distance two) appeared in 1972 to compute the total electron energy of the alternant hydrocarbons. But after that, for a long period, these are not studied by researchers. Recently, Ali and Trinajstic [Mol. Inform. 37(2018), 1-7] restudied the Zagreb connection indices and reported that the Zagreb connection indices comparatively to the classical Zagreb indices provide the better absolute value of the correlation coefficient for the thirteen physicochemical properties of the octane isomers (all these tested values have been taken from the website http://www.moleculardescriptors.eu). In this paper, we compute the general results in the form of exact formulae \& upper bounds of the second Zagreb connection index and modified first Zagreb connection index for the resultant graphs which are obtained by applying operations of corona, Cartesian, and lexicographic product. At the end, some applications of the obtained results for particular chemical structures such as alkanes, cycloalkanes, linear polynomial chain, carbon nanotubes, fence, and closed fence are presented. In addition, a comparison between exact and computed values of the aforesaid Zagreb indices is also included.

\section{Introduction}

Graph theory has provided a variety of useful tools in which one of the best tools is a topological index (TI). Molecules and molecular compounds are often modeled by molecular graphs. The topological indices (TIs) predict hydrocarbon, physicochemical, and structural properties of the molecular graphs such as critical temperature, ZE-isomerism, chirality, solubility, molecular mass, and connectivity, see [1-4]. Medical behaviours of the drugs, crystallin materials, and nanomaterials which are very important for chemical and pharmaceutical industries are also studied by TIs, see [5-8]. Todeschini et al. [9] also reported that TIs are widely used in the study of quantitative structure-activity relationships (QSARs) and quantitative structure-property relationships
(QSPRs). These relationships play a vital role in the subject of cheminformatics, see [9-13].

TIs have been divided into different classes, but degreebased are studied more, see $[1,4,7,14,15,16]$. Gutman and Trinajstić [17] investigated the correlation value between the total $\pi$-electron energy and the structure of a molecule using the first Zagreb index. Gutman et al. [18] developed their work and established another TI for molecular structures called the second Zagreb index. After that, many extended works have been appeared on these invariants. For more study, we refer to $[9,10,19,20]$. Another TI was studied by Gutman and Trinajstić in the same paper [17], but there was not more attention on this index by other researchers up to 2017. Ali and Trinajstić [21] restudied this TI and renamed it as the modified first Zagreb connection index (ZCI). They 
also reported that it has more precise values of the correlation coefficients of various octane isomers. Du et al. [22], Ducoffe et al. [23], and Shao et al. [24] determined extremal alkanes and cycloalkanes under different conditions using this ZCI. Zhu et al. [25] established the lower bound by using the modified first ZCI of trees in terms of their order and maximum degree. Tang et al. [26] computed the first and second (ZCI) and modified first (ZCI) of the $S$-sum graphs.

A simple graph can be molded into a chemical structure by using some operations. First of all, Graovac and Pisanski [27] computed different results of the Wiener index using product based on operations. So, many chemical graphs can be generated by using simple graphs based on operations such as alkane $\left(\mathrm{C}_{3} \mathrm{H}_{6}\right)$ is the corona product of $P_{3} \& N_{2}$, a type of cycloalkanes is cyclohexane $\left(\mathrm{C}_{6} \mathrm{H}_{12}\right)$ that is the corona product of $C_{6} \& N_{2}$, a polynomial chain and nanotube $\left(\mathrm{TUC}_{4}(m, n)\right)$ are the Cartesian product of $P_{m} \& P_{2}$ and $C_{m} \& P_{2}$, and a fence and closed fence are the lexicographic product of $P_{m} \& P_{2}$ and $C_{m} \& P_{2}$. Up till now, many results of the various TIs have been presented under different molecular graphs based on operations, see [26, 28-38].

In this paper, we compute the second ZCI and modified first ZCI of the resultant graphs which are obtained by applying various operations of corona product, Cartesian product, and lexicographic product (composition) in the form of exact formulae and upper bounds. The rest of the paper is settled as Section 2 represents the preliminary definitions and results, Section 3 covers the general results of molecular graphs based on operations, and Section 4 includes the applications and conclusion.

\section{Preliminaries}

Let $Q=(V(Q), E(Q))$ be a simple and connected molecular graph with a vertex set $V(Q)$ and an edge set $E(Q) \subseteq V(Q) \times V(Q)$. A null graph $(N)$ has at least two vertices and no edge. It becomes a trivial graph $K_{1}$ if it has exactly one vertex and no edge. Todesehini et al. [9] defined ${ }^{k} f_{Q}(b)=\left|{ }^{k} N_{Q}(b)\right|$ for ${ }^{k} N_{Q}(b)=\{a \in V(Q): d(a, b)=k\}$ such that ${ }^{1} f_{Q}(b)=d_{G}(b)$ and ${ }^{2} f_{Q}(b)=\tau_{G}(b)$ are called the degree and connection number of the vertex $b \in Q$. Now, throughout the paper, we assume that $Q_{1}$ and $Q_{2}$ are two connected graphs such that $\left|V\left(Q_{1}\right)\right|=n_{1},\left|V\left(Q_{2}\right)\right|=n_{2}$, $\left|E\left(Q_{1}\right)\right|=e_{1}$, and $\left|E\left(Q_{2}\right)\right|=e_{2}$.

Alkanes are the simplest organic compounds containing a single bound between carbon atoms. They are also called hydrocarbon compounds. Its simple and Lewis structures are shown in Figure 1.

The some examples of alkanes are methane $\left(\mathrm{CH}_{4}\right)$, ethane $\left(\mathrm{H}_{3} \mathrm{C}-\mathrm{CH}_{3}\right)$, and propane $\left(\mathrm{H}_{3} \mathrm{C}-\mathrm{CH}_{2}-\mathrm{CH}_{3}\right)$, and their Lewis structures are shown in Figure 2.

Cycloalkanes are cyclic organic compounds containing the closed chain of carbon atoms. In other words, a cycloalkane is arranged into a chemical structure obtained a single ring (sometime side chains may be attached), and all of the carbon-carbon bonds are single. These are classified into two classes as homocyclic and heterocyclic compounds. If cyclo-organic compounds containing between carbons [ 1 to 5], [6 to 10], and [11 to on wards] are called small, mediam, and large cyclo organic compounds, respectively. Cycloalkanes are the isomers of alkene, e.g., $\mathrm{C}_{3} \mathrm{H}_{6}$ is used as the same chemical formula of cyclopropane as well as propene. The general formula of cycloalkanes is $\mathrm{C}_{n} \mathrm{H}_{2(n)}$. The sets of \{Cyclopropane, cyclobutane, cyclopentane, cyclohexane etc. $\&$ \{pyrol, thiophene etc. $\}$ are examples of cycloalkanes that are classified by homocyclic and heterocyclic compounds, respectively. Furthermore, the following Lewis structures of these cycloalkanes are shown in Figure 3.

Definition 1. For a graph $Q$, the first Zagreb index $\left(M_{1}(Q)\right)$, second Zagreb index $\left(M_{2}(Q)\right)$, and their coindices are defined as

$$
\begin{aligned}
& M_{1}(Q)=\sum_{b \in V(Q)}\left[d_{Q}(b)\right]^{2}=\sum_{a b \in E(Q)}\left[d_{Q}(a)+d_{Q}(b)\right], \\
& M_{2}(Q)=\sum_{a b \in E(Q)}\left[d_{Q}(a) \times d_{Q}(b)\right], \\
& \overline{M_{1}}(Q)=\sum_{a b \notin E(Q)}\left[d_{Q}(a)+d_{Q}(b)\right], \\
& \overline{M_{2}}(Q)=\sum_{a b \notin E(Q)}\left[d_{Q}(a) \times d_{Q}(b)\right] .
\end{aligned}
$$

Gutman et al. $[17,18,39]$ defined the different degreebased TIs which are frequently used in the studies of QSPR and QSAR [40-43]. Corresponding to these degree-based TIs, the connection-based TIs are defined in Definition 2. For further studies of connection-based TIs, see [21, 22, 44].

Definition 2. For a graph $Q$, the first Zagreb connection index $\left(Z C_{1}(Q)\right)$, second Zagreb connection index $\left(Z C_{2}(Q)\right)$, and modified first Zagreb connection index $\left(Z C_{1}^{*}(Q)\right)$ are defined as

$$
\begin{aligned}
& Z C_{1}(Q)=\sum_{b \in V(Q)}\left[\tau_{Q}(b)\right]^{2}, \\
& Z C_{2}(Q)=\sum_{a b \in E(Q)}\left[\tau_{Q}(a) \times \tau_{Q}(b)\right], \\
& Z C_{1}^{*}(Q)=\sum_{b \in V(Q)} d_{Q}(b) \tau_{Q}(b)=\sum_{a b \in E(Q)}\left[\tau_{Q}(a)+\tau_{Q}(b)\right] .
\end{aligned}
$$

Definition 3. The corona product $Q_{1} \circ Q_{2}$ of two graphs $Q_{1}$ and $Q_{2}$ is obtained by taking one copy of $Q_{1}$ and $n_{1}$ copies of $Q_{2}$ (i.e., $\left.\left\{Q_{2}^{i}: 1 \leq i \leq n_{1}\right\}\right)$ and then by joining each vertex of the $i^{\text {th }}$ copy of $Q_{2}$ to the $i^{\text {th }}$ vertex of one copy of $Q_{1}$, where $1 \leq i \leq n_{1}$. Also, a number of vertex set and edge set are defined as: $\left|V\left(Q_{1} \circ Q_{2}\right)\right|=n_{1} n_{2}+n_{1}$ and $\left|E\left(Q_{1} \circ Q_{2}\right)\right|=e_{1}$ $+n_{1} e_{2}+n_{1} n_{2}$. For more detail, see Figure 4 . 


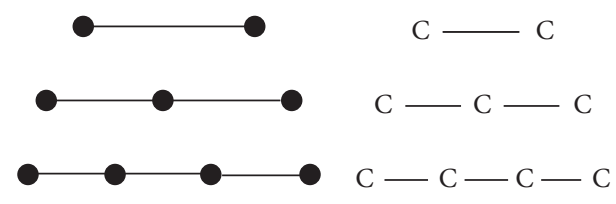

(a) (b)

Figure 1: (a) Simple structure of alkanes $P_{2}, P_{3}$, and $P_{4}$, respectively, and (b) Lewis structure of alkanes $P_{2}, P_{3}$, and $P_{4}$, respectively.

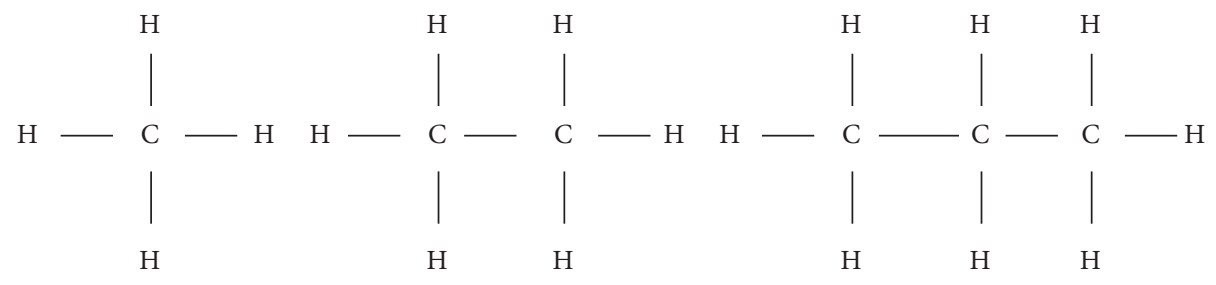

(a)

(b)

(c)

Figure 2: Lewis structure of (a) methane, (b) ethane and (c) propane.<smiles>C1CC1</smiles><smiles>C1CCC1</smiles><smiles>c1cc[nH]c1</smiles><smiles></smiles><smiles>C1C2CC12</smiles><smiles>C1CCCCC1</smiles>

(a)

(b)

Figure 3: (a) The set of Lewis structure of cyclopropane, cyclobutane, cyclopentane, and cyclohexane, (b) the set of Lewis structure of pyrrole and thiophene.
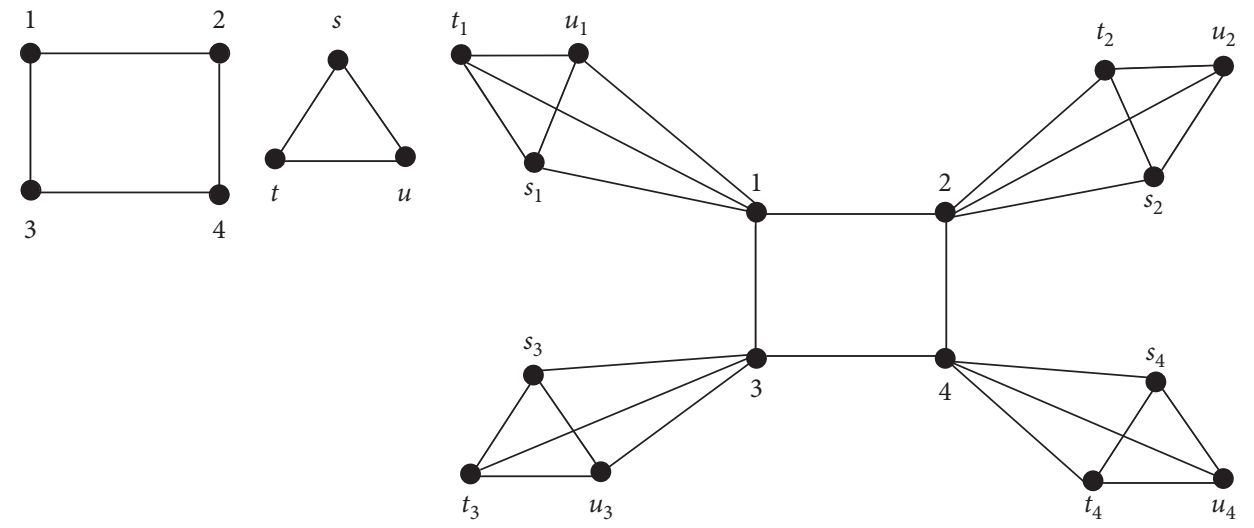

(a)

(b)

(c)

Figure 4: (a) $Q_{1} \cong C_{4}$, (b) $Q_{2} \cong C_{3}$, and (c) CoronaProduct $\left(C_{4} \circ C_{3}\right)$. 
Definition 4. The Cartesian product $\left(Q_{1} \odot Q_{2}\right)$ and lexicographic product or composition $\left(Q_{1}\left[Q_{2}\right]\right)$ of two graphs $Q_{1}$ and $Q_{2}$ are obtained by taking the vertex set $V\left(Q_{1} \times Q_{2}\right)=$ $V\left(Q_{1}\right) \times V\left(Q_{2}\right)$ and the edge set $E\left(Q_{1} \times Q_{2}\right)=$ $\left[\left\{\left(a_{1}, b_{1}\right)\left(a_{2}, b_{2}\right)\right.\right.$ where $\left.\left.\left(a_{1}, b_{1}\right),\left(a_{2}, b_{2}\right) \in V\left(Q_{1}\right) \times V\left(Q_{2}\right)\right\}\right]$, where with conditions

(i) either $\left[a_{1}=a_{2} \in V\left(Q_{1}\right) \wedge b_{1} b_{2} \in E \quad\left(Q_{2}\right)\right] \quad$ or $\left[b_{1}=b_{2} \in V\left(Q_{2}\right) \wedge a_{1} a_{2} \in E\left(Q_{1}\right)\right]$,

(ii) either $\left[a_{1}=a_{2} \in V\left(Q_{1}\right) \wedge b_{1} b_{2} \in E\left(Q_{2}\right)\right]$ or $\left[b_{1}, b_{2}\right.$ $\left.\in V\left(Q_{2}\right) \wedge a_{1} a_{2} \in E\left(Q_{1}\right)\right]$, respectively.

For more detail, see Figures 5 and 6.

Lemma 1 (see [45]). Let $Q$ be a connected graph and $\bar{Q}$ be its complement. Then, (i) $\sum_{b \in V(Q)} d_{Q}(b)=2 e$, (ii) $d_{\bar{Q}}(b)=$ $(n-1)-d_{Q}(b)$, and (iii) $M_{1}(\bar{Q})=M_{1}(Q)-4$, where $|V(Q)|=n$ and $|E(Q)|=e$.

Lemma 2 (see [46]). Let $Q$ be a connected graph with $n$ vertices and e edges. Then, $\tau_{Q}(a)+d_{Q}(a) \leq \sum_{b \in N_{Q}(a)}\left(d_{Q}(b)\right)$, where equality holds if and only if $Q$ is a $\left\{C_{3}, C_{4}\right\}$ - free graph.

Lemma 3 (see [26]). Let $Q$ be a connected and $\left\{C_{3}, C_{4}\right\}$-free graph with $n$ vertices and e edges. Then, $\sum_{a \in V(Q)} \tau_{Q}(a)=$ $M_{1}(Q)-2 e$.

\section{Main Results}

This section consists on the main results.
Theorem 1. Let $Q_{1}$ and $Q_{2}$ be two connected and $\left\{C_{3}, C_{4}\right\}-$ free graphs. Then, $Z C_{2}$ and $Z C_{1}^{*}$ of the corona product of $Q_{1}$ and $\mathrm{Q}_{2}$ are as follows:

(a) $Z C_{2}\left(G_{1} \circ Q_{2}\right)=n_{2} Z C_{1}^{*}\left(Q_{1}\right)+Z C_{2}\left(Q_{1}\right)+n_{2}^{2} M_{2}\left(Q_{1}\right)$

$$
\begin{aligned}
& +n_{1} M_{2}\left(Q_{2}\right)+\left(n_{2}^{2}+e_{2}\right) M_{1}\left(Q_{1}\right) \\
& -n_{1}\left(n_{2}-1\right) M_{1}\left(Q_{2}\right)+n_{1}\left(n_{2}-1\right)^{2} e_{2} \\
& +2 e_{1}\left[2\left(n_{2}-1\right) e_{2}-M_{1}\left(Q_{2}\right)\right] \\
& +\left[n_{2}\left(n_{2}-1\right)-2 e_{2}\right]\left[M_{1}\left(G_{1}\right)-2 e_{1}\right] \\
& +2 e_{1}\left[n_{2}^{2}\left(n_{2}-1\right)-2 n_{2} e_{2}\right] \\
& +n_{2} \sum_{a b \in E\left(Q_{1}\right)}\left[d_{Q_{1}}(a) \tau_{Q_{1}}(b)+d_{Q_{1}}(b) \tau_{Q_{1}}(a)\right] .
\end{aligned}
$$

(b) $Z C_{1}^{*}\left(Q_{1} \circ Q_{2}\right)=Z C_{1}^{*}\left(Q_{1}\right)+2 n_{2} M_{1}\left(Q_{1}\right)-n_{1} M_{1}\left(Q_{2}\right)$

$$
+2 n_{1} e_{2}\left(n_{2}-2\right)+2 n_{2}^{2} e_{1}+n_{1} n_{2}\left(n_{2}-1\right)+4 e_{1} e_{2} .
$$

Proof

(a) If for any $b \in V\left(Q_{1} \circ Q_{2}\right)$ either $b \in V\left(Q_{1}\right)$ or $b \in V\left(Q_{2}^{i}\right)$, where $1 \leq i \leq n_{1}$ and

(i) Case I: if $b \in V\left(Q_{1}\right)$, then $\tau_{Q_{1} \circ Q_{2}}(b)=\tau_{Q_{1}}(b)$ $+n_{2} d_{Q_{1}}(b)$.

(ii) Case II: if $b \in V\left(Q_{2}^{i}\right)$, then $\tau_{Q_{1} \circ Q_{2}}(b)=\left(n_{2}-1\right)$ $-d_{Q_{2}^{i}}(b)+d_{Q_{1}}\left(b_{i}\right)$

$$
\begin{aligned}
Z C_{2}\left(Q_{1} \circ Q_{2}\right)= & \sum_{a b \in E\left(Q_{1} \circ Q_{2}\right)}\left[\tau_{\left(Q_{1} \circ Q_{2}\right)}(a) \times \tau_{\left(Q_{1} \circ Q_{2}\right)}(b)\right] \\
= & \sum_{\substack{a b \in E\left(Q_{1} \circ Q_{2}\right) \\
a, b \in V\left(Q_{1}\right)}}\left[\tau_{Q_{1}}(a) \times \tau_{Q_{1}}(b)\right]+\sum_{\substack{a b \in E\left(Q_{1} \circ Q_{2}\right) \\
a, b \in V\left(Q_{2}\right)}}\left[\tau_{Q_{2}}(a) \times \tau_{Q_{2}}(b)\right]+\sum_{\substack{a b \in E\left(Q_{1} \circ Q_{2}\right) \\
a \in V\left(Q_{1}\right) \wedge b \in V\left(Q_{2}\right)}}\left[\tau_{Q_{1}}(a) \times \tau_{Q_{2}}(b)\right] .
\end{aligned}
$$

Take

$$
\begin{aligned}
\sum_{u v \in E\left(Q_{1} Q_{2}\right)}\left[\tau_{Q_{1}}(a) \times \tau_{Q_{1}}(b)\right] & =\sum_{a b \in E\left(Q_{1}\right)}\left[\left\{\tau_{Q_{1}}(a)+n_{2} d_{Q_{1}}(a)\right\} \times\left\{\tau_{Q_{1}}(b)+n_{2} d_{Q_{1}}(b)\right\}\right] \\
& =\sum_{a b \in V\left(Q_{1}\right)}\left[\tau_{Q_{1}}(a) \tau_{Q_{1}}(b)+n_{2} d_{Q_{1}}(b) \tau_{Q_{1}}(a)+n_{2} d_{Q_{1}}(a) \tau_{Q_{1}}(b)+n_{2}^{2} d_{Q_{1}}(a) d_{Q_{1}}(b)\right] \\
& =Z C_{2}\left(Q_{1}\right)+n_{2}^{2} M_{2}\left(Q_{1}\right)+n_{2} \sum_{a b \in E\left(Q_{1}\right)}\left[d_{Q_{1}}(a) \tau_{Q_{1}}(b)+d_{Q_{1}}(b) \tau_{Q_{1}}(a)\right] .
\end{aligned}
$$



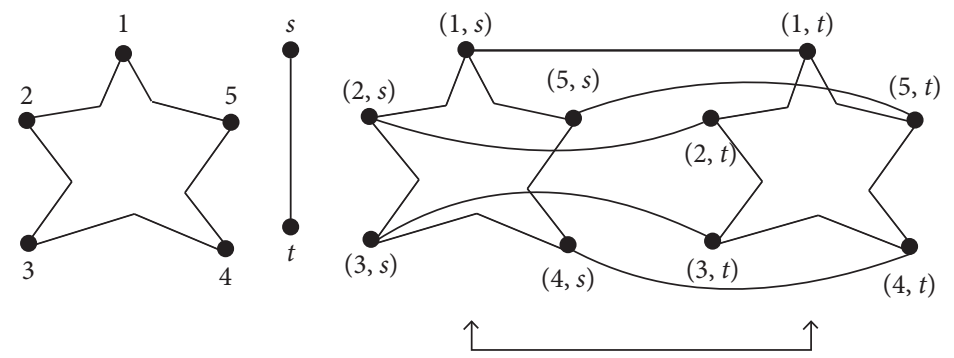

(a)

(b)

(c)

Figure 5: (a) $Q_{1} \cong C_{5}$, (b) $Q_{2} \cong P_{2}$, and (c) CartesianProduct $\left(C_{5} \odot P_{2}\right)$.
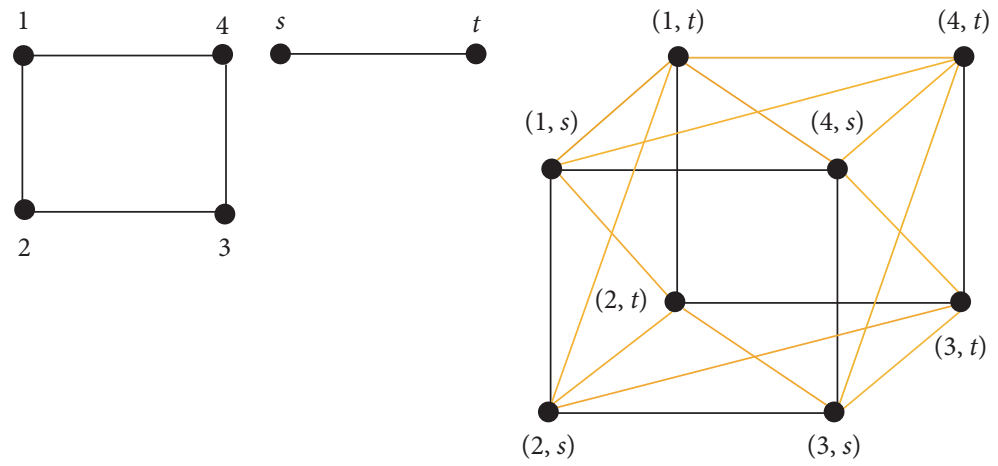

(a)

(b)

(c)

FIgURe 6: (a) $Q_{1} \cong C_{4}$, (b) $Q_{2} \cong P_{2}$, and (c) LexicographicProduct $\left(\mathrm{C}_{4}\left[\mathrm{P}_{2}\right]\right)$.

Also take

$$
\begin{aligned}
& \sum_{\substack{a b \in E\left(Q_{1} Q_{2}\right) \\
a, b \in V\left(Q_{2}\right)}}\left[\tau_{Q_{2}}(a) \times \tau_{Q_{2}}(b)\right] \\
= & \sum_{i=1}^{n_{1}} \sum_{a b \in E\left(Q_{2}^{i}\right)}\left[\left\{\left(n_{2}-1\right)-d_{Q_{2}^{i}}(a)+d_{Q_{1}}\left(b_{i}\right)\right\} \times\left\{\left(n_{2}-1\right)-d_{Q_{2}^{i}}(b)+d_{Q_{1}}\left(b_{i}\right)\right\}\right] \\
= & \sum_{i=1}^{n_{1}} \sum_{a b \in E\left(Q_{2}^{i}\right)}\left[\left(n_{2}-1\right)^{2}-\left(n_{2}-1\right)\left\{d_{Q_{2}^{i}}(a)+d_{Q_{2}^{i}}(b)\right\}+2\left(n_{2}-1\right) d_{Q_{1}}\left(b_{i}\right)+d_{Q_{2}^{i}}(u) d_{Q_{2}^{i}}(b)-d_{Q_{1}}\left(b_{i}\right) d_{Q_{2}^{i}}(a)\right. \\
& \left.-d_{Q_{1}}\left(b_{i}\right) d_{Q_{2}^{i}}(b)+d_{Q_{1}}^{2}\left(b_{i}\right)\right] \\
= & n_{1}\left(n_{2}-1\right)^{2} e_{2}-n_{1}\left(n_{2}-1\right) M_{1}\left(Q_{2}\right)+2 e_{1}\left[2\left(n_{2}-1\right) e_{2}-M_{1}\left(Q_{2}\right)\right]+n_{1} M_{2}\left(Q_{2}\right)+e_{2} M_{1}\left(Q_{1}\right) .
\end{aligned}
$$

Similarly,

$$
\begin{aligned}
& \sum_{a b \in E\left(Q_{1}^{\circ} Q_{2}\right)}\left[\tau_{Q_{1}}(a) \times \tau_{Q_{2}}(b)\right] \\
& a \in V\left(Q_{1}\right) \wedge b \in V\left(Q_{2}\right) \\
&= \sum_{i=1}^{n_{1}} \sum_{b \in V\left(Q_{2}^{i}\right)}\left[\left\{\tau_{Q_{1}}\left(a_{i}\right)+n_{2} d_{Q_{1}}\left(a_{i}\right)\right\} \times\left\{\left(n_{2}-1\right)-d_{Q_{2}^{i}}(b)+d_{Q_{1}}\left(a_{i}\right)\right\}\right] \\
&= {\left[n_{2}\left(n_{2}-1\right)-2 e_{2}\right]+\left[M_{1}\left(Q_{1}\right)-2 e_{1}\right]+n_{2} Z C_{1}^{*}\left(Q_{1}\right)+2 e_{1}\left[n_{2}^{2}\left(n_{2}-1\right)-2 n_{2} e_{2}\right]+n_{2}^{2} M_{1}\left(Q_{1}\right) . }
\end{aligned}
$$


Consequently,

$$
\begin{aligned}
Z C_{2}\left(Q_{1} \circ Q_{2}\right)= & n_{2} Z C_{1}^{*}\left(Q_{1}\right)+Z C_{2}\left(Q_{1}\right)+n_{2}^{2} M_{2}\left(Q_{1}\right)+n_{1} M_{2}\left(Q_{2}\right)+\left(n_{2}^{2}+e_{2}\right) M_{1}\left(Q_{1}\right) \\
& -n_{1}\left(n_{2}-1\right) M_{1}\left(Q_{2}\right)+n_{1}\left(n_{2}-1\right)^{2} e_{2}+2 e_{1}\left[2\left(n_{2}-1\right) e_{2}-M_{1}\left(Q_{2}\right)\right]+\left[n_{2}\left(n_{2}-1\right)-2 e_{2}\right]\left[M_{1}\left(G_{1}\right)-2 e_{1}\right] \\
& +2 e_{1}\left[n_{2}^{2}\left(n_{2}-1\right)-2 n_{2} e_{2}\right]+n_{2} \sum_{a b \in E\left(Q_{1}\right)}\left[d_{Q_{1}}(a) \tau_{Q_{1}}(b)+d_{Q_{1}}(b) \tau_{Q_{1}}(a)\right] .
\end{aligned}
$$

(b)

$$
\begin{aligned}
Z C_{1}^{*}\left(Q_{1} \circ Q_{2}\right) & =\sum_{a b \in E\left(Q_{1} \circ Q_{2}\right)}\left[\tau_{\left(Q_{1} \circ Q_{2}\right)}(a)+\tau_{\left(Q_{1} \circ Q_{2}\right)}(b)\right] \\
& =\sum_{\substack{a b \in E\left(Q_{1} \circ Q_{2}\right) \\
a, b \in V\left(Q_{1}\right)}}\left[\tau_{Q_{1}}(a)+\tau_{Q_{1}}(b)\right]+\sum_{\substack{a b \in E\left(G_{1} \circ G_{2}\right) \\
a, b \in V\left(Q_{2}\right)}}\left[\tau_{Q_{2}}(a)+\tau_{Q_{2}}(b)\right]+\sum_{\substack{a b \in E \\
a \in V\left(Q_{1} Q_{2}\right) \wedge b \in V\left(Q_{2}\right)}}\left[\tau_{Q_{1}}(a)+\tau_{Q_{2}}(b)\right] .
\end{aligned}
$$

Take

$$
\sum_{\substack{a b \in E\left(Q_{1} Q_{2}\right) \\ a, b \in V\left(Q_{1}\right)}}\left[\tau_{Q_{1}}(a)+\tau_{Q_{1}}(b)\right]=\sum_{a b \in E\left(Q_{1}\right)}\left[\left\{\tau_{Q_{1}}(a)+n_{2} d_{Q_{1}}(a)\right\}+\left\{\tau_{Q_{1}}(b)+n_{2} d_{Q_{1}}(b)\right\}\right]==Z C_{1}^{*}\left(Q_{1}\right)+n_{2} M_{1}\left(Q_{1}\right)
$$

Also take

$$
\begin{aligned}
& \sum_{\substack{a b \in E\left(Q_{1} Q_{2}\right) \\
a, b \in V\left(Q_{2}\right)}}\left[\tau_{\mathrm{Q}_{2}}(a)+\tau_{\mathrm{Q}_{2}}(b)\right] \\
= & \sum_{i=1}^{n_{1}} \sum_{a b \in E\left(Q_{2}^{i}\right)}\left[\left\{\left(n_{2}-1\right)-d_{\mathrm{Q}_{2}^{i}}(u)+d_{\mathrm{Q}_{1}}\left(b_{i}\right)\right\}+\left\{\left(n_{2}-1\right)-d_{\mathrm{Q}_{2}^{i}}(b)+d_{\mathrm{Q}_{1}}\left(b_{i}\right)\right\}\right] \\
= & 2 n_{1}\left(n_{2}-1\right) e_{2}-n_{1} M_{1}\left(Q_{2}\right)+4 e_{1} e_{2} .
\end{aligned}
$$

Similarly,

$$
\sum_{\substack{a b \in E\left(Q_{1} \circ Q_{2}\right) \\ a \in V\left(Q_{1}\right) \wedge b \in V\left(Q_{2}\right)}}\left[\tau_{Q_{1}}(a)+\tau_{Q_{2}}(b)\right]=n_{2}\left[M_{1}\left(Q_{1}\right)-2 e_{1}\right]+2 n_{2}^{2} e_{1}+n_{1} n_{2}\left(n_{2}-1\right)-2 n_{1} e_{2}+2 n_{2} e_{1}
$$

Consequently,

$$
Z C_{1}^{*}\left(Q_{1} \circ Q_{2}\right)=Z C_{1}^{*}\left(Q_{1}\right)+2 n_{2} M_{1}\left(Q_{1}\right)-n_{1} M_{1}\left(Q_{2}\right)+2 n_{1} e_{2}\left(n_{2}-2\right)+2 n_{2}^{2} e_{1}+n_{1} n_{2}\left(n_{2}-1\right)+4 e_{1} e_{2}
$$

Theorem 2. Let $Q_{1}$ and $Q_{2}$ be two connected and $\left\{C_{3}, C_{4}\right\}-$ free graphs. Then, $Z C_{2}$ and $Z C_{1}^{*}$ of the Cartesian product of $Q_{1}$ and $Q_{2}$ are as follows: 
(a) $Z C_{2}\left(Q_{1} \odot Q_{2}\right)=2\left[M_{1}\left(Q_{2}\right)-e_{2}\right] Z C_{1}^{*}\left(Q_{1}\right)+2\left[M_{1}\left(Q_{1}\right)-e_{1}\right] Z C_{1}^{*}\left(Q_{2}\right)+n_{2} Z C_{2}\left(Q_{1}\right)$

$$
\begin{aligned}
& +n_{1} Z C_{2}\left(Q_{2}\right)+e_{2} Z C_{1}\left(Q_{1}\right)+e_{1} Z C_{1}\left(Q_{2}\right)+M_{1}\left(Q_{1}\right) M_{2}\left(Q_{2}\right)+M_{1}\left(Q_{2}\right) M_{2}\left(Q_{1}\right) \\
& +2 e_{2} \sum_{a_{1} a_{2} \in E\left(Q_{1}\right)}\left[d_{Q_{1}}\left(u_{1}\right) \tau_{Q_{1}}\left(a_{2}\right)+d_{Q_{1}}\left(a_{2}\right) \tau_{Q_{1}}\left(a_{1}\right)\right] \\
& +2 e_{1} \sum_{b_{1} b_{2} \in E\left(Q_{2}\right)}\left[d_{Q_{2}}\left(b_{1}\right) \tau_{Q_{2}}\left(b_{2}\right)+d_{Q_{2}}\left(b_{2}\right) \tau_{Q_{2}}\left(b_{1}\right)\right]
\end{aligned}
$$

(b) $Z C_{1}^{*}\left(Q_{1} \odot Q_{2}\right)=n_{2} Z C_{1}^{*}\left(Q_{1}\right)+n_{1} Z C_{1}^{*}\left(Q_{2}\right)+4 e_{2} M_{1}\left(Q_{1}\right)+4 e_{1} M_{1}\left(Q_{2}\right)-8 e_{1} e_{2}$.

Proof

(a) For $a \in V\left(Q_{1}\right), b \in V\left(Q_{2}\right)$, and $(a, b) \in V\left(Q_{1} \odot Q_{2}\right)$, we have

$$
\begin{aligned}
\tau_{\mathrm{Q}_{1} \odot \mathrm{Q}_{2}}(a, b) & =\tau_{\mathrm{Q}_{1}}(a)+d_{\mathrm{Q}_{1}}(a) d_{\mathrm{Q}_{2}}(b)+\tau_{\mathrm{Q}_{2}}(b), \\
Z C_{2}\left(\mathrm{Q}_{1} \odot Q_{2}\right) & =\sum_{\left(a_{1}, b_{1}\right)\left(a_{2}, b_{2}\right) \in E\left(\mathrm{Q}_{1} \odot \mathrm{Q}_{2}\right)}\left[\tau_{\mathrm{Q}_{1} \odot \mathrm{Q}_{2}}\left(a_{1}, b_{1}\right) \times \tau_{\mathrm{Q}_{1} \odot \mathrm{Q}_{2}}\left(a_{2}, b_{2}\right)\right] \\
& =\sum_{a \in V\left(\mathrm{Q}_{1}\right)} \sum_{b_{1} b_{2} \in E\left(\mathrm{Q}_{2}\right)}\left[\tau_{\mathrm{Q}_{1} \odot \mathrm{Q}_{2}}\left(a, b_{1}\right) \times \tau_{\mathrm{Q}_{1} \odot \mathrm{Q}_{2}}\left(a, b_{2}\right)\right]+\sum_{b \in V\left(\mathrm{Q}_{2}\right)} \sum_{a_{1} a_{2} \in E\left(\mathrm{Q}_{1}\right)}\left[\tau_{\mathrm{Q}_{1} \odot \mathrm{Q}_{2}}\left(a_{1}, b\right) \times \tau_{\mathrm{Q}_{1} \odot \mathrm{Q}_{2}}\left(a_{2}, b\right)\right] .
\end{aligned}
$$

Take

$$
\begin{aligned}
& \sum_{a \in V\left(\mathrm{Q}_{1}\right)} \sum_{b_{1} b_{2} \in E\left(\mathrm{Q}_{2}\right)}\left[\tau_{\mathrm{Q}_{1} \odot \mathrm{Q}_{2}}\left(a, b_{1}\right) \times \tau_{\mathrm{Q}_{1} \odot \mathrm{Q}_{2}}\left(a, b_{2}\right)\right] \\
& =\sum_{a \in V\left(\mathrm{Q}_{1}\right)} \sum_{b_{1} b_{2} \in E\left(\mathrm{Q}_{2}\right)}\left[\left\{\tau_{\mathrm{Q}_{1}}(a)+d_{\mathrm{Q}_{1}}(a) d_{\mathrm{Q}_{2}}\left(b_{1}\right)+\tau_{\mathrm{Q}_{2}}\left(b_{1}\right)\right\} \times\left\{\tau_{\mathrm{Q}_{1}}(a)+d_{\mathrm{Q}_{1}}(a) d_{\mathrm{Q}_{2}}\left(b_{2}\right)+\tau_{\mathrm{Q}_{2}}\left(b_{2}\right)\right\}\right] \\
& =e_{2} Z C_{1}\left(Q_{1}\right)+M_{1}\left(\mathrm{Q}_{2}\right) Z C_{1}^{*}\left(Q_{1}\right)+Z C_{1}^{*}\left(Q_{2}\right)\left[M_{1}\left(Q_{1}\right)-2 e_{1}\right]+M_{1}\left(Q_{1}\right) M_{2}\left(Q_{2}\right)+n_{1} Z C_{2}\left(Q_{2}\right) \\
& \quad+2 e_{1} \sum_{b_{1} b_{2} E\left(\mathrm{Q}_{2}\right)}\left[d_{\mathrm{Q}_{2}}\left(b_{1}\right) \tau_{\mathrm{Q}_{2}}\left(b_{2}\right)+d_{\mathrm{Q}_{2}}\left(b_{2}\right) \tau_{\mathrm{Q}_{2}}\left(b_{1}\right)\right] .
\end{aligned}
$$

Similarly,

$$
\begin{aligned}
& \sum_{b \in V\left(Q_{2}\right)} \sum_{a_{1} a_{2} \in E\left(Q_{1}\right)}\left[\tau_{Q_{1} \odot Q_{2}}\left(a_{1}, b\right) \times \tau_{\mathrm{Q}_{1} \odot Q_{2}}\left(a_{2}, b\right)\right] \\
& =n_{2} Z C_{2}\left(Q_{1}\right)+Z C_{1}^{*}\left(Q_{1}\right)\left[M_{1}\left(Q_{2}\right)-2 e_{2}\right]+2 e_{2} \sum_{a_{1} a_{2} \in E\left(Q_{1}\right)}\left[d_{Q_{1}}\left(a_{1}\right) \tau_{Q_{1}}\left(a_{2}\right)+d_{Q_{1}}\left(a_{2}\right) \tau_{Q_{1}}\left(a_{1}\right)\right] \\
& \quad+M_{1}\left(Q_{2}\right) M_{2}\left(Q_{1}\right)+M_{1}\left(Q_{1}\right) Z C_{1}^{*}\left(Q_{2}\right)+e_{1} Z C_{1}^{*}\left(Q_{2}\right) .
\end{aligned}
$$

Consequently,

$$
\begin{aligned}
& Z C_{2}\left(Q_{1} \odot Q_{2}\right)=2\left[M_{1}\left(Q_{2}\right)-e_{2}\right] Z C_{1}^{*}\left(Q_{1}\right)+2\left[M_{1}\left(Q_{1}\right)-e_{1}\right] Z C_{1}^{*}\left(Q_{2}\right)+n_{2} Z C_{2}\left(Q_{1}\right) \\
& +n_{1} Z C_{2}\left(G_{2}\right)+e_{2} Z C_{1}\left(Q_{1}\right)+e_{1} Z C_{1}\left(Q_{2}\right)+M_{1}\left(Q_{1}\right) M_{2}\left(Q_{2}\right)+M_{1}\left(Q_{2}\right) M_{2}\left(Q_{1}\right)+2 e_{2} \\
& \quad \sum_{a_{1} a_{2} \in E\left(Q_{1}\right)}\left[d_{Q_{1}}\left(u_{1}\right) \tau_{Q_{1}}\left(a_{2}\right)+d_{Q_{1}}\left(a_{2}\right) \tau_{Q_{1}}\left(a_{1}\right)\right]+2 e_{1} \sum_{b_{1} b_{2} \in E\left(Q_{2}\right)}\left[d_{Q_{2}}\left(b_{1}\right) \tau_{Q_{2}}\left(b_{2}\right)+d_{Q_{2}}\left(b_{2}\right) \tau_{Q_{2}}\left(b_{1}\right)\right] .
\end{aligned}
$$


(b) Consider

$$
\begin{aligned}
Z C_{1}^{*}\left(Q_{1} \odot Q_{2}\right) & =\sum_{\left(a_{1}, b_{1}\right)\left(a_{2}, b_{2}\right) \in E\left(Q_{1} \odot Q_{2}\right)}\left[\tau_{Q_{1} \odot Q_{2}}\left(a_{1}, b_{1}\right)+\tau_{Q_{1} \odot Q_{2}}\left(a_{2}, b_{2}\right)\right] \\
& =\sum_{a \in V\left(Q_{1}\right)} \sum_{b_{1} b_{2} \in E\left(Q_{2}\right)}\left[\tau_{Q_{1} \odot Q_{2}}\left(a, b_{1}\right)+\tau_{Q_{1} \odot Q_{2}}\left(a, b_{2}\right)\right]+\sum_{b \in V\left(Q_{2}\right)} \sum_{a_{1} a_{2} \in E\left(Q_{1}\right)}\left[\tau_{Q_{1} \odot Q_{2}}\left(a_{1}, b\right)+\tau_{Q_{1} \odot Q_{2}}\left(a_{2}, b\right)\right]
\end{aligned}
$$

Take

$$
\begin{aligned}
& \sum_{a \in V\left(Q_{1}\right)} \sum_{b_{1} b_{2} \in E\left(Q_{2}\right)}\left[\tau_{Q_{1} \odot Q_{2}}\left(a, b_{1}\right)+\tau_{Q_{1} \odot Q_{2}}\left(a, b_{2}\right)\right] \\
& =\sum_{a \in V\left(Q_{1}\right)} \sum_{b_{1} b_{2} \in E\left(Q_{2}\right)}\left[\left\{\tau_{Q_{1}}(a)+d_{Q_{1}}(a) d_{Q_{2}}\left(b_{1}\right)+\tau_{Q_{2}}\left(b_{1}\right)\right\}+\left\{\tau_{Q_{1}}(a)+d_{Q_{1}}(a) d_{Q_{2}}\left(b_{2}\right)+\tau_{Q_{2}}\left(b_{2}\right)\right\}\right] \\
& =2 e_{2}\left[M_{1}\left(Q_{1}\right)-2 e_{1}\right]+2 e_{1} M_{1}\left(Q_{2}\right)+n_{1} Z C_{1}^{*}\left(Q_{2}\right) .
\end{aligned}
$$

Similarly,

$$
\begin{aligned}
& \sum_{b \in V\left(Q_{2}\right)} \sum_{a_{1} a_{2} \in E\left(Q_{1}\right)}\left[\tau_{Q_{1} \odot Q_{2}}\left(a_{1}, b\right)+\tau_{Q_{1} \odot Q_{2}}\left(a_{2}, b\right)\right] \\
& =n_{2} Z C_{1}^{*}\left(Q_{1}\right)+2 e_{2} M_{1}\left(Q_{1}\right)+2 e_{1} M_{1}\left(Q_{2}\right)-4 e_{1} e_{2} .
\end{aligned}
$$

Consequently,

$$
Z C_{1}^{*}\left(Q_{1} \odot Q_{2}\right)=n_{2} Z C_{1}^{*}\left(Q_{1}\right)+n_{1} Z C_{1}^{*}\left(Q_{2}\right)+4 e_{2} M_{1}\left(Q_{1}\right)+4 e_{1} M_{1}\left(Q_{2}\right)-8 e_{1} e_{2}
$$

Theorem 3. Let $Q_{1}$ and $Q_{2}$ be two connected graphs. Then, $Z C_{2}$ and $Z C_{1}^{*}$ of the composition (or lexicographic product) of $Q_{1}$ and $Q_{2}$ are as follows:

(a) $\mathrm{ZC}_{2}\left(Q_{1}\left[Q_{2}\right]\right) \leq n_{2}\left[n_{2}\left(n_{2}-1\right)-4 e_{2}+2 n_{2} e_{2}+n_{2} \delta_{2}-\delta_{2}\right] \mathrm{ZC}_{1}^{*}\left(Q_{1}\right)+n_{2}^{2}\left(n_{2}+\delta_{2}+2 e_{2}\right) \mathrm{ZC}_{2}\left(Q_{1}\right)$

$$
\begin{aligned}
& +n_{2}^{2} e_{2} \mathrm{ZC}_{1}\left(Q_{1}\right)+\left(n_{1}-n_{1} n_{2}-2 n_{2} e_{1}+3 e_{1}\right) M_{1}\left(Q_{2}\right)+\left(n_{1}+2 e_{1}\right) M_{2}\left(Q_{2}\right)+\left[2 n_{2}\left(n_{2}-1\right) e_{2}-n_{2} M_{1}\left(Q_{2}\right)\right] \\
& {\left[M_{1}\left(Q_{1}\right)-2 e_{1}\right]+\left(n_{2}-1\right)\left[n_{1} e_{2}\left(n_{2}-1\right)+e_{1} e_{2}\left(n_{2}-2\right)+\delta_{2}\right.} \\
& \left.\left(n_{2}+n_{2} e_{2}-e_{1}-1\right)\right]-\left(n_{2}-1\right) e_{1} \overline{M_{1}}\left(Q_{2}\right)+e_{1} \overline{M_{2}}\left(Q_{2}\right) \\
& \quad-2 n_{2} \sum_{a_{1} a_{2} \in E\left(Q_{1}\right)} \sum_{b_{1} b_{2} \in E\left(Q_{2}\right)}\left[d_{Q_{2}}\left(b_{1}\right) \tau_{Q_{1}}\left(a_{2}\right)+d_{Q_{2}}\left(b_{2}\right) \tau_{Q_{1}}\left(a_{1}\right)\right] \\
& -n_{2} \sum_{a_{1} a_{2} \in E\left(Q_{1}\right) b_{1} b_{2} \notin E\left(Q_{2}\right)}\left[d_{Q_{2}}\left(b_{1}\right) \tau_{Q_{1}}\left(a_{2}\right)+d_{Q_{2}}\left(b_{2}\right) \tau_{Q_{1}}\left(a_{1}\right)\right]
\end{aligned}
$$

(b) $\mathrm{ZC}_{1}^{*}\left(Q_{1}\left[Q_{2}\right]\right) \leq n_{2}\left(n_{2}+\delta_{2}+2 e_{2}\right) \mathrm{ZC}_{1}^{*}\left(Q_{1}\right)+2 n_{2} e_{2} M_{1}\left(Q_{1}\right)-\left(n_{1}+2 e_{1}\right) M_{1}\left(Q_{2}\right)$

$$
+\left(n_{2}-1\right)\left(2 n_{1} e_{2}+2 n_{2} e_{1}+2 \delta_{2} e_{1}+4 e_{1} e_{2}\right)-4\left(n_{2}+1\right) e_{1} e_{2}-e_{1} \overline{M_{1}}\left(Q_{2}\right) .
$$


Proof

(a) For $a \in V\left(Q_{1}\right), b \in V\left(Q_{2}\right)$, and $(a, b) \in V\left(Q_{1}\left[Q_{2}\right]\right)$, we have

$$
\begin{aligned}
\tau_{Q_{1}\left[Q_{2}\right]}(a, b)= & n_{2} \tau_{Q_{1}}(a)+d \overline{Q_{2}}(b)=n_{2} \tau_{Q_{1}}(a)+\left(n_{2}-1\right)-d_{Q_{2}}(b) \\
Z C_{2}\left(Q_{1}\left[Q_{2}\right]\right)= & \sum_{\left(a_{1}, b_{1}\right)\left(a_{2}, b_{2}\right) \in E\left(Q_{1}\left[Q_{2}\right]\right)}\left[\tau_{Q_{1}\left[Q_{2}\right]}\left(a_{1}, b_{1}\right) \times \tau_{Q_{1}\left[Q_{2}\right]}\left(a_{2}, b_{2}\right)\right] \\
= & \sum_{a \in V\left(Q_{1}\right)} \sum_{b_{1} b_{2} \in E\left(Q_{2}\right)}\left[\tau_{Q_{1}\left[Q_{2}\right]}\left(a, b_{1}\right) \times \tau_{Q_{1}\left[Q_{2}\right]}\left(a, b_{2}\right)\right]+\sum_{b \in V\left(Q_{2}\right)} \sum_{a_{1} a_{2} \in E\left(Q_{1}\right)}\left[\tau_{Q_{1}\left[Q_{2}\right]}\left(a_{1}, b\right) \times \tau_{Q_{1}\left[Q_{2}\right]}\left(a_{2}, b\right)\right] \\
& +\sum_{a_{1} a_{2} \in E\left(Q_{1}\right) b_{1} b_{2} \in E\left(Q_{2}\right)}\left[\tau_{Q_{1}\left[Q_{2}\right]}\left(a_{1}, b_{1}\right) \times \tau_{Q_{1}\left[Q_{2}\right]}\left(a_{2}, b_{2}\right)\right] \\
& +\sum_{a_{1} a_{2} \in E\left(Q_{1}\right) b_{1} b_{2} \notin E\left(Q_{2}\right)}\left[\tau_{Q_{1}\left[Q_{2}\right]}\left(a_{1}, b_{1}\right) \times \tau_{Q_{1}\left[Q_{2}\right]}\left(a_{2}, b_{2}\right)\right] .
\end{aligned}
$$

Take

$$
\begin{aligned}
& \sum_{a \in V\left(Q_{1}\right)} \sum_{b_{1} b_{2} \in E\left(Q_{2}\right)}\left[\tau_{Q_{1}\left[Q_{2}\right]}\left(a, b_{1}\right) \times \tau_{Q_{1}\left[Q_{2}\right]}\left(a, b_{2}\right)\right] \\
& =\sum_{a \in V\left(Q_{1}\right)} \sum_{b_{1} b_{2} \in E\left(Q_{2}\right)}\left[\left\{n_{2} \tau_{Q_{1}}(a)+\left(n_{2}-1\right)-d_{Q_{2}}\left(b_{1}\right)\right\} \times\left\{n_{2} \tau_{Q_{1}}(a)+\left(n_{2}-1\right)-d_{Q_{2}}\left(b_{2}\right)\right\}\right] \\
& =n_{2}^{2} e_{2} Z C_{1}\left(Q_{1}\right)+\left[2 n_{2}\left(n_{2}-1\right) e_{2}-n_{2} M_{1}\left(Q_{2}\right)\right]\left[M_{1}\left(Q_{1}\right)-2 e_{1}\right]+n_{1}\left(n_{2}-1\right)^{2} e_{2}-n_{1}\left(n_{2}-1\right) M_{1}\left(Q_{2}\right)+n_{1} M_{2}\left(Q_{2}\right)
\end{aligned}
$$

Also take

$$
\begin{aligned}
& \sum_{a_{1} a_{2} \in E\left(Q_{1}\right)} \sum_{b_{1} b_{2} \notin E\left(Q_{2}\right)}\left[\tau_{Q_{1}\left[Q_{2}\right]}\left(a_{1}, b_{1}\right) \times \tau_{Q_{1}\left[Q_{2}\right]}\left(a_{2}, b_{2}\right)\right] \\
\leq & \sum_{a_{1} a_{2} \in E\left(Q_{1}\right)} \sum_{b_{1} b_{2} \notin E\left(Q_{2}\right)}\left[\left\{n_{2} \tau_{Q_{1}}\left(a_{1}\right)+\left(n_{2}-1\right)-d_{Q_{2}}\left(b_{1}\right)\right\} \times\left\{n_{2} \tau_{Q_{1}}\left(a_{2}\right)+\left(n_{2}-1\right)-d_{Q_{2}}\left(b_{2}\right)\right\}\right] \\
= & \sum_{a_{1} a_{2} \in E\left(Q_{1}\right)} \sum_{b_{1} b_{2} \notin E\left(Q_{2}\right)}\left[n_{2}^{2} \tau_{Q_{1}}\left(a_{1}\right) \tau_{Q_{1}}\left(a_{2}\right)+n_{2}\left(n_{2}-1\right)\left\{\tau_{Q_{1}}\left(a_{1}\right)+\tau_{Q_{1}}\left(a_{2}\right)\right\}-n_{2}\left\{d_{Q_{2}}\left(b_{1}\right) \tau_{Q_{1}}\left(a_{2}\right)+d_{Q_{2}}\left(b_{2}\right) \tau_{Q_{1}}\left(a_{1}\right)\right\}\right. \\
+ & \left.\left(n_{2}-1\right)^{2}-\left(n_{2}-1\right)\left\{d_{Q_{2}}\left(b_{1}\right)+d_{Q_{2}}\left(b_{2}\right)\right\}+d_{Q_{2}}\left(b_{1}\right) d_{Q_{2}}\left(b_{2}\right)\right] .
\end{aligned}
$$

Let's suppose that

$$
\begin{aligned}
\sum_{b_{1} b_{2} \notin E\left(Q_{2}\right)}= & n_{2}\left(n_{2}-1\right)-2 e_{2}=\delta_{2} \\
= & n_{2}^{2} \delta_{2} Z C_{2}\left(Q_{1}\right)+n_{2}\left(n_{2}-1\right) \delta_{2} Z C_{1}^{*}\left(Q_{1}\right)-n_{2} \sum_{a_{1} a_{2} \in E\left(Q_{1}\right)} \sum_{b_{1} b_{2} \notin E\left(Q_{2}\right)}\left[d_{Q_{2}}\left(b_{1}\right) \tau_{Q_{1}}\left(a_{2}\right)+d_{Q_{2}}\left(b_{2}\right) \tau_{Q_{1}}\left(a_{1}\right)\right] \\
& +\left(n_{2}-1\right)^{2} \delta_{2} e_{1}-\left(n_{2}-1\right) e_{1} \overline{M_{1}}\left(Q_{2}\right)+e_{1} \overline{M_{2}}\left(Q_{2}\right) .
\end{aligned}
$$


Similarly,

$$
\begin{aligned}
& \sum_{b \in V\left(Q_{2}\right)} \sum_{a_{1} a_{2} \in E\left(Q_{1}\right)}\left[\tau_{Q_{1}\left[Q_{2}\right]}\left(a_{1}, b\right) \times \tau_{Q_{1}\left[Q_{2}\right]}\left(a_{2}, b\right)\right] \\
= & n_{2}\left[n_{2}\left(n_{2}-1\right)-2 e_{2}\right] Z C_{1}^{*}\left(Q_{1}\right)+n_{2}^{3} Z C_{2}\left(Q_{1}\right)+e_{1} M_{1}\left(Q_{2}\right)+\left(n_{2}-1\right) e_{1}\left[n_{2}\left(n_{2}-1\right)-4 e_{2}\right] . \\
a_{1} a_{2} \in & E\left(Q_{1}\right) \sum_{a_{1} a_{2} \in E\left(Q_{1}\right)} \sum_{b_{1} b_{2} \in E\left(Q_{2}\right)}\left[\tau_{Q_{1}\left[Q_{2}\right]}\left(a_{1}, b_{1}\right) \times \tau_{Q_{1}\left[Q_{2}\right]}\left(a_{2}, b_{2}\right)\right] \\
= & \sum_{a_{1} a_{2} \in E\left(Q_{1}\right)} \sum_{b_{1} b_{2} \in E\left(Q_{2}\right)}\left[\left\{n_{2} \tau_{Q_{1}}\left(a_{1}\right)+\left(n_{2}-1\right)-d_{Q_{2}}\left(b_{1}\right)\right\} \times\left\{n_{2} \tau_{Q_{1}}\left(a_{2}\right)+\left(n_{2}-1\right)-d_{Q_{2}}\left(b_{2}\right)\right\}\right] \\
= & 2 n_{2}^{2} e_{2} Z C_{2}\left(Q_{1}\right)+2 n_{2}\left(n_{2}-1\right) e_{2} Z C_{1}^{*}\left(Q_{1}\right)-2 n_{2} \sum_{a_{1} a_{2} \in E\left(Q_{1}\right) b_{1} b_{2} \in E\left(Q_{2}\right)}\left[d_{Q_{2}}\left(b_{1}\right) \tau_{Q_{1}}\left(a_{2}\right)+d_{Q_{2}}\left(b_{2}\right) \tau_{Q_{1}}\left(a_{1}\right)\right] \\
& +2\left(n_{2}-1\right)^{2} e_{1} e_{2}-2\left(n_{2}-1\right) e_{1} M_{1}\left(Q_{2}\right)+2 e_{1} M_{2}\left(Q_{2}\right) .
\end{aligned}
$$

Consequently,

$$
\begin{aligned}
Z C_{2}\left(Q_{1}\left[Q_{2}\right]\right) \leq & n_{2}\left[n_{2}\left(n_{2}-1\right)-4 e_{2}+2 n_{2} e_{2}+n_{2} \delta_{2}-\delta_{2}\right] Z C_{1}^{*}\left(Q_{1}\right)+n_{2}^{2}\left(n_{2}+\delta_{2}+2 e_{2}\right) Z C_{2}\left(Q_{1}\right) \\
& +n_{2}^{2} e_{2} Z C_{1}\left(Q_{1}\right)+\left(n_{1}-n_{1} n_{2}-2 n_{2} e_{1}+3 e_{1}\right) M_{1}\left(Q_{2}\right)+\left(n_{1}+2 e_{1}\right) M_{2}\left(Q_{2}\right)+\left[2 n_{2}\left(n_{2}-1\right) e_{2}-n_{2} M_{1}\left(Q_{2}\right)\right] \\
& {\left[M_{1}\left(Q_{1}\right)-2 e_{1}\right]+\left(n_{2}-1\right)\left[n_{1} e_{2}\left(n_{2}-1\right)+e_{1} e_{2}\left(n_{2}-2\right)+\delta_{2}\left(n_{2}+n_{2} e_{2}-e_{1}-1\right)\right], } \\
& -\left(n_{2}-1\right) e_{1} \overline{M_{1}}\left(Q_{2}\right)+e_{1} \overline{M_{2}}\left(Q_{2}\right)-2 n_{2} \sum_{a_{1} a_{2} \in E\left(Q_{1}\right)} \sum_{b_{1} b_{2} \in E\left(Q_{2}\right)}\left[d_{Q_{2}}\left(b_{1}\right) \tau_{Q_{1}}\left(a_{2}\right)+d_{Q_{2}}\left(b_{2}\right) \tau_{Q_{1}}\left(a_{1}\right)\right] \\
& -n_{2} \sum_{a_{1} a_{2} \in E\left(Q_{1}\right) b_{1} b_{2} \notin E\left(Q_{2}\right)}\left[d_{Q_{2}}\left(b_{1}\right) \tau_{Q_{1}}\left(a_{2}\right)+d_{Q_{2}}\left(b_{2}\right) \tau_{Q_{1}}\left(a_{1}\right)\right] .
\end{aligned}
$$

(b)

$$
\begin{aligned}
Z C_{1}^{*}\left(Q_{1}\left[Q_{2}\right]\right)= & \sum_{\left(a_{1}, b_{1}\right)\left(a_{2}, b_{2}\right) \in E\left(Q_{1}\left[Q_{2}\right]\right)}\left[\tau_{Q_{1}\left[Q_{2}\right]}\left(a_{1}, b_{1}\right)+\tau_{Q_{1}\left[Q_{2}\right]}\left(a_{2}, b_{2}\right)\right] \\
= & \sum_{a \in V\left(Q_{1}\right)} \sum_{b_{1} b_{2} \in E\left(Q_{2}\right)}\left[\tau_{Q_{1}\left[Q_{2}\right]}\left(a, b_{1}\right)+\tau_{Q_{1}\left[Q_{2}\right]}\left(a, b_{2}\right)\right] \\
& +\sum_{b \in V\left(Q_{2}\right)} \sum_{a_{1} a_{2} \in E\left(Q_{1}\right)}\left[\tau_{Q_{1}\left[Q_{2}\right]}\left(a_{1}, b\right)+\tau_{Q_{1}\left[Q_{2}\right]}\left(a_{2}, b\right)\right] \\
& +\sum_{a_{1} a_{2} \in E\left(Q_{1}\right) b_{1} b_{2} \in E\left(Q_{2}\right)}\left[\tau_{Q_{1}\left[Q_{2}\right]}\left(a_{1}, b_{1}\right)+\tau_{Q_{1}\left[Q_{2}\right]}\left(a_{2}, b_{2}\right)\right] \\
& +\sum_{a_{1} a_{2} \in E\left(Q_{1}\right) b_{1} b_{2} \notin E\left(Q_{2}\right)}\left[\tau_{Q_{1}\left[Q_{2}\right]}\left(a_{1}, b_{1}\right)+\tau_{Q_{1}\left[Q_{2}\right]}\left(a_{2}, b_{2}\right)\right] .
\end{aligned}
$$

Take

$$
\begin{aligned}
& \sum_{a \in V\left(Q_{1}\right)} \sum_{b_{1} b_{2} \in E\left(Q_{2}\right)}\left[\tau_{Q_{1}\left[G_{2}\right]}\left(a, b_{1}\right)+\tau_{Q_{1}\left[Q_{2}\right]}\left(a, b_{2}\right)\right] \\
& =\sum_{a \in V\left(Q_{1}\right)} \sum_{b_{1} b_{2} \in E\left(Q_{2}\right)}\left[\left\{n_{2} \tau_{Q_{1}}(a)+\left(n_{2}-1\right)-d_{Q_{2}}\left(b_{1}\right)\right\}+\left\{n_{2} \tau_{Q_{1}}(a)+\left(n_{2}-1\right)-d_{Q_{2}}\left(b_{2}\right)\right\}\right. \\
& =2 n_{2} e_{2}\left[M_{1}\left(Q_{1}\right)-2 e_{1}\right]+2 n_{1}\left(n_{2}-1\right) e_{2}-n_{1} M_{1}\left(Q_{2}\right) .
\end{aligned}
$$




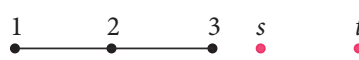

(a)

(b)

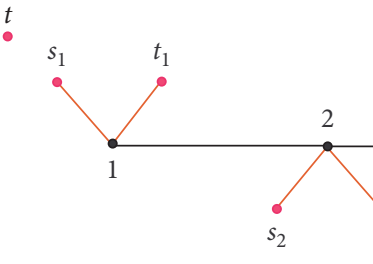

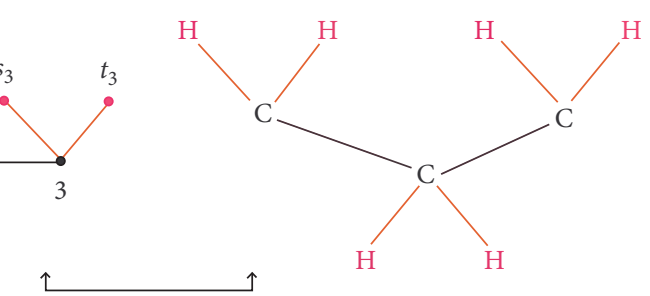

(c)

Figure 7: (a) $Q_{1} \cong P_{3}$, (b) $Q_{2} \cong N_{2}$, and (c) alkane $\left(P_{3} \circ N_{2} \cong \mathrm{C}_{3} \mathrm{H}_{6}\right)$.

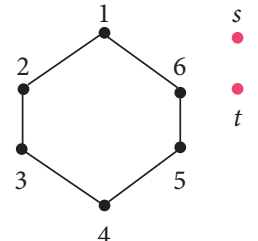

(a)

(b)

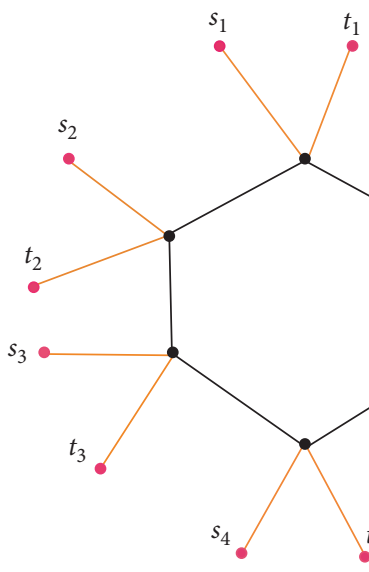

Figure 8: (a) $Q_{1} \cong C_{6}$, (b) $Q_{2} \cong N_{2}$, and (c) cyclohexane $\left(C_{6} \circ N_{2} \cong \mathrm{C}_{6} \mathrm{H}_{12}\right)$.
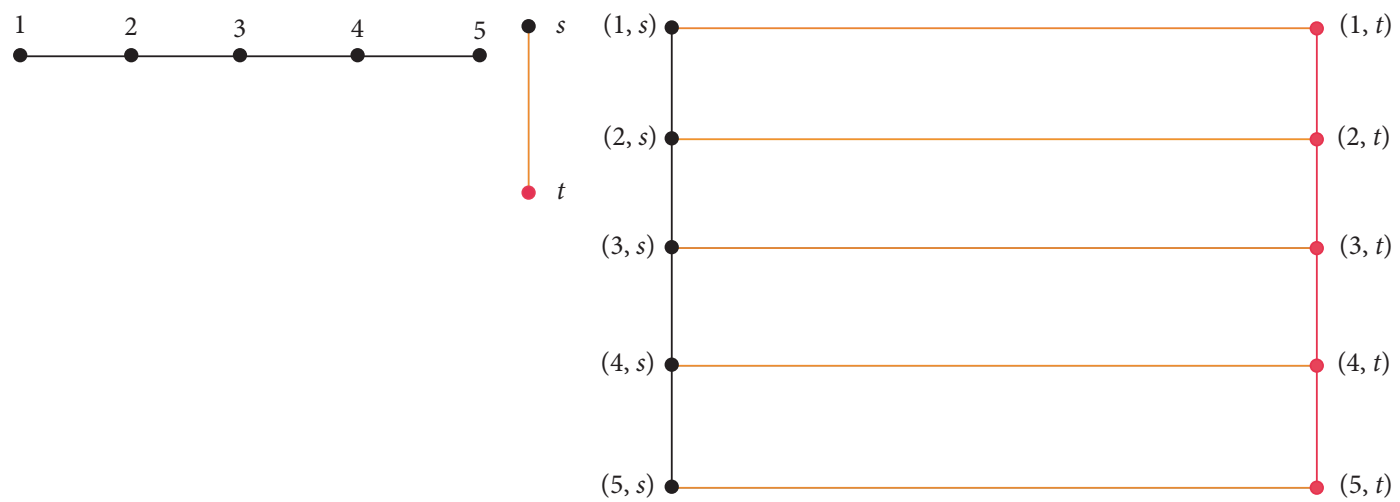

(a)

(b)

(c)

Figure 9: (a) $Q_{1} \cong P_{5}$, (b) $Q_{2} \cong P_{2}$, and (c) $\left(P_{5} \odot P_{2}\right)$.

Also take

$$
\begin{aligned}
& \sum_{a_{1} a_{2} \in E\left(Q_{1}\right)} \sum_{b_{1} b_{2} \notin E\left(Q_{2}\right)}\left[\tau_{Q_{1}\left[Q_{2}\right]}\left(a_{1}, b_{1}\right)+\tau_{Q_{1}\left[Q_{2}\right]}\left(a_{2}, b_{2}\right)\right] \\
\leq & \sum_{a_{1} a_{2} \in E\left(Q_{1}\right)} \sum_{b_{1} b_{2} \notin E\left(Q_{2}\right)}\left[\left\{n_{2} \tau_{Q_{1}}\left(a_{1}\right)+\left(n_{2}-1\right)-d_{Q_{2}}\left(b_{1}\right)\right\}+\left\{n_{2} \tau_{Q_{1}}\left(a_{2}\right)+\left(n_{2}-1\right)-d_{Q_{2}}\left(b_{2}\right)\right\}\right] \\
= & n_{2} \delta_{2} Z C_{1}^{*}\left(Q_{1}\right)+2\left(n_{2}-1\right) \delta_{2} e_{1}-e_{1} \overline{M_{1}}\left(Q_{2}\right) .
\end{aligned}
$$




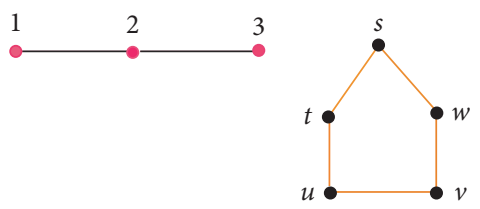

(a)

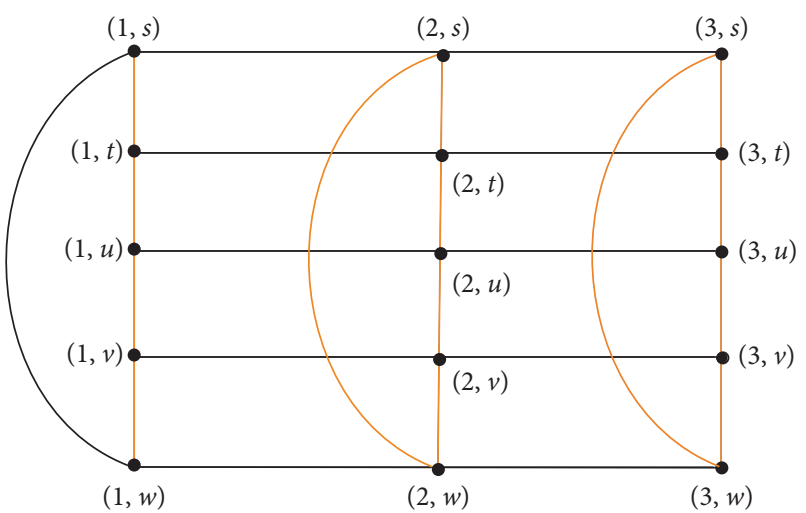

(c)

Figure 10: (a) $Q_{1} \cong P_{3}$, (b) $Q_{2} \cong C_{5}$, and (c) carbon nanotube $\left(\operatorname{TUC}_{4}(m, n)\right)\left(P_{3} \odot C_{5}\right)$.
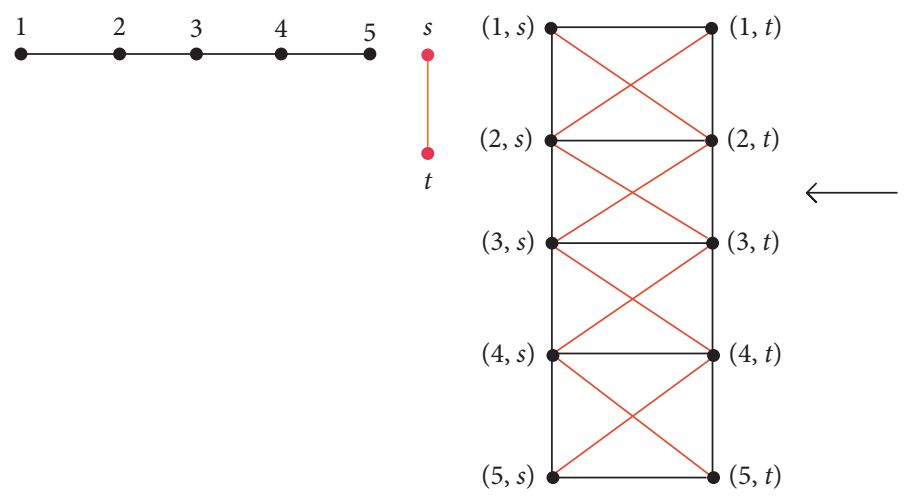

(a)

(b)

(c)

Figure 11: (a) $Q_{1} \cong P_{5}$, (b) $Q_{2} \cong P_{2}$, and (c) fence $\left(P_{5}\left[P_{2}\right]\right)$.
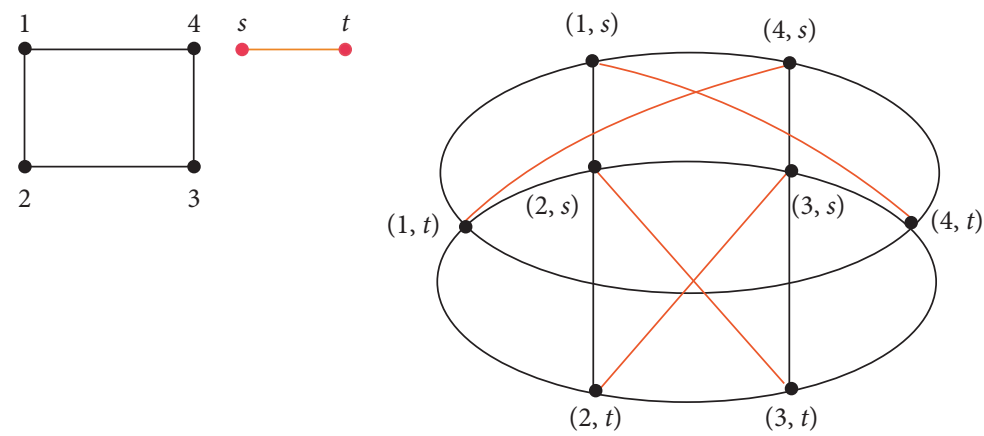

(a)

(b)

(c)

Figure 12: (a) $Q_{1} \cong C_{4}$, (b) $Q_{2} \cong P_{2}$, and (c) closed fence $\left(C_{4}\left[P_{2}\right]\right)$.

Similarly,

$$
\begin{aligned}
& \sum_{b \in V\left(Q_{2}\right)} \sum_{a_{1} a_{2} \in E\left(Q_{1}\right)}\left[\tau_{Q_{1}\left[Q_{2}\right]}\left(a_{1}, b\right)+\tau_{Q_{1}\left[Q_{2}\right]}\left(a_{2}, b\right)\right] \\
= & n_{2}^{2} Z C_{1}^{*}\left(Q_{1}\right)+2 n_{2}\left(n_{2}-1\right) e_{1}-4 e_{1} e_{2}, \sum_{a_{1} a_{2} \in E\left(Q_{1}\right)} \sum_{b_{1} b_{2} \in E\left(Q_{2}\right)}\left[\tau_{Q_{1}\left[Q_{2}\right]}\left(a_{1}, b_{1}\right)+\tau_{Q_{1}\left[Q_{2}\right]}\left(a_{2}, b_{2}\right)\right] \\
= & 2 \sum_{a_{1} a_{2} \in E\left(Q_{1}\right) b_{1} b_{2} \in E\left(Q_{2}\right)}\left[\left\{n_{2} \tau_{Q_{1}}\left(a_{1}\right)+\left(n_{2}-1\right)-d_{Q_{2}}\left(b_{1}\right)\right\}+\left\{n_{2} \tau_{Q_{1}}\left(a_{2}\right)+\left(n_{2}-1\right)-d_{Q_{2}}\left(b_{2}\right)\right\}\right] \\
= & 2 n_{2} e_{2} Z C_{1}^{*}\left(Q_{1}\right)+4\left(n_{2}-1\right) e_{1} e_{2}-2 e_{1} M_{1}\left(Q_{2}\right) .
\end{aligned}
$$


Consequently,

$$
\begin{aligned}
Z C_{1}^{*}\left(Q_{1}\left[Q_{2}\right]\right) \leq & n_{2}\left(n_{2}+\delta_{2}+2 e_{2}\right) Z C_{1}^{*}\left(Q_{1}\right)+2 n_{2} e_{2} M_{1}\left(Q_{1}\right)-\left(n_{1}+2 e_{1}\right) M_{1}\left(Q_{2}\right)+\left(n_{2}-1\right)\left(2 n_{1} e_{2}+2 n_{2} e_{1}+2 \delta_{2} e_{1}+4 e_{1} e_{2}\right) \\
& -4\left(n_{2}+1\right) e_{1} e_{2}-e_{1} \overline{M_{1}}\left(Q_{2}\right) .
\end{aligned}
$$

\section{Applications and Conclusion}

In this section, we present some applications of the obtained results for particular chemical structures such as alkanes (see Figure 7), cycloalkanes (see Figure 8), linear polynomial chain (see Figure 9), carbon nanotubes (see Figure 10), fence (see Figure 11), and closed fence (see Figure 12). We also give the both exact and computed values of the obtained results for the aforesaid particular chemical structures to develop an easy understanding. Let $N_{2}$ be a null graph, $P_{2}, P_{3}$, and $P_{5}$ be three particular alkanes called by paths, and $C_{4}, C_{5}$, and $C_{6}$ be cycles.

\subsection{Corona Product}

Example 1. Alkane $\left(\mathrm{C}_{3} \mathrm{H}_{6}\right)$.

(i) Exact value of $Z C_{2}\left(P_{3} \circ N_{2}\right)=72$,

(ii) Exact value of $Z C_{1}^{*}\left(P_{3} \circ N_{2}\right)=48$,

(iii) Computed value of $Z C_{2}\left(P_{3} \circ N_{2}\right)=72$,

(iv) Computed value of $Z C_{1}^{*}\left(P_{3} \circ N_{2}\right)=48$.

Example 2. Cyclohexane $\left(\mathrm{C}_{6} \mathrm{H}_{12}\right)$.

(i) Exact value of $Z C_{2}\left(C_{6} \circ N_{2}\right)=432$,

(ii) Exact value of $Z C_{1}^{*}\left(C_{6} \circ N_{2}\right)=180$,

(iii) Computed value of $Z C_{2}\left(C_{6} \circ N_{2}\right)=432$,

(iv) Computed value of $Z C_{1}^{*}\left(C_{6} \circ N_{2}\right)=180$.

\subsection{Cartesian Product}

Example 3. Polynomial chain.

(i) Exact value of $Z C_{2}\left(P_{5} \odot P_{2}\right)=114$,

(ii) Exact value of $Z C_{1}^{*}\left(P_{5} \odot P_{2}\right)=76$,

(iii) Computed value of $Z C_{2}\left(P_{5} \odot P_{2}\right)=114$,

(iv) Computed value of $Z C_{1}^{*}\left(P_{5} \odot P_{2}\right)=76$.

Example 4. Carbon nanotube $\left(\mathrm{TUC}_{4}(m, n)\right)$.

(i) Exact value of $Z C_{2}\left(P_{3} \odot C_{5}\right)=730$,

(ii) Exact value of $Z C_{1}^{*}\left(P_{3} \odot C_{5}\right)=270$,

(iii) Computed value of $Z C_{2}\left(P_{3} \odot C_{5}\right)=730$,

(iv) Computed value of $Z C_{1}^{*}\left(P_{3} \odot C_{5}\right)=270$.

\subsection{Lexicographic Product}

Example 5. Fence $\left(P_{5}\left[P_{2}\right]\right)$.

(i) Exact value of $Z C_{2}\left(P_{5}\left[P_{2}\right]\right)=128$,

(ii) Exact value of $Z C_{1}^{*}\left(P_{5}\left[P_{2}\right]\right)=104$,

(iii) Computed value of $Z C_{2}\left(P_{3}\left[P_{3}\right]\right)=128$,

(iv) Computed value of $Z C_{1}^{*}\left(P_{5}\left[P_{2}\right]\right)=104$.

Example 6. Closed fence $\left(C_{4}\left[P_{2}\right]\right)$.

(i) Exact value of $Z C_{2}\left(C_{4}\left[P_{2}\right]\right)=72$,

(ii) Exact value of $Z C_{1}^{*}\left(C_{4}\left[P_{2}\right]\right)=72$,

(iii) Computed value of $Z C_{2}\left(C_{4}\left[P_{2}\right]\right) \leq 80$,

(iv) Computed value of $Z C_{1}^{*}\left(C_{4}\left[P_{2}\right]\right) \leq 88$.

In this paper, we have computed the general results related to the second ZCI and modified first ZCI of the resultant graphs which are obtained with the help of various operations of product on graphs such as corona product, Cartesian product, and lexicographic product (composition). The obtained results also illustrated with the help of particular class of molecular graphs. However, the problem is still open to compute the ZCI of the molecular graphs under the different operations of subdivision, addition and product, etc.

\section{Data Availability}

All the data are included within this paper. However, the reader may contact the corresponding author for more details of the data.

\section{Conflicts of Interest}

The authors have no conflicts of interest.

\section{Acknowledgments}

This work was supported in part by the National Natural Science Foundation of China (grant no 61833005) and in part by the Jiangsu Provincial Key Laboratory of Networked Collective Intelligence (grant no BM2017002).

\section{References}

[1] A. R. Matamala and E. Estrada, "Generalised topological indices: optimisation methodology and physico-chemical interpretation," Chemical Physics Letters, vol. 410, no. 4-6, pp. 343-347, 2005. 
[2] S. Nikolic, I. M. Tolic, and N. Trinajstic, "On the complexity of molecular graphs," MATCH Communications in Mathematical and in Computer Chemistry, vol. 40, 2000.

[3] S. Nikolic and N. Trinajstic, "The Zagreb indices 30 years after," Croatica Chemica Acta, vol. 76, pp. 113-124, 2003.

[4] G. Rücker and C. Rücker, "On topological indices, boiling points, and cycloalkanes," Journal of Chemical Information and Computer Sciences, vol. 39, no. 5, pp. 788-802, 1999.

[5] H. Gonzalez-Diaz, S. Vilar, L. Santana, and E. Uriarte, "Medicinal chemistry and bioinformatics-current trends in drugs discovery with networks topological indices," Current Topics in Medicinal Chemistry, vol. 7, no. 10, pp. 1015-1029, 2007.

[6] R. Gozalbes, J. Doucet, and F. Derouin, "Application of topological descriptors in QSAR and drug design: history and new trends," Current Drug Target -Infectious Disorders, vol. 2, no. 1, pp. 93-102, 2002.

[7] L. H. Hall and L. B. Kier, Molecular Connectivity in Chemistry and Drug Research, Academic Press, Boston, MA, USA, 1976.

[8] W. Yan, B.-Y. Yang, and Y.-N. Yeh, "The behavior of Wiener indices and polynomials of graphs under five graph decorations," Applied Mathematics Letters, vol. 20, no. 3, pp. 290-295, 2007.

[9] R. Todeschini, V. Consonni, R. Mannhold, H. Kubinyi, and H. Timmerman, Handbook of Molecular Descriptors, WileyVCH, Weinheim, Germany, 2002.

[10] I. Gutman and O. Polansky, Mathematical Concepts in Organic Chemistry, Springer-Verlag, Berlin, Germany, 1986.

[11] M. Iswarya, R. Raja, G. Rajchakit, J. Cao, J. Alzabut, and C. Huang, "Existence, uniqueness and exponential stability of periodic solution for discrete-time delayed BAM neural networks based on coincidence degree theory and graph theoretic method," Mathematics, vol. 7, no. 11, p. 1055, 2019.

[12] Y. Cao, L. Zhang, C. Li, and M. Z. Q. Chen, "Observer-based consensus tracking of nonlinear agents in hybrid varying directed topology," IEEE Transactions on Cybernetics, vol. 47, no. 8, pp. 2212-2222, 2017.

[13] I. Manickam, R. Raja, G. Rajchakit, J. Cao, J. Alzabut, and C. Huang, "A perspective on graph theory-based stability analysis of impulsive stochastic recurrent neural networks with time-varying delays," Advances in Difference Equations, vol. 2019, no. 1, p. 502, 2019.

[14] I. Gutman, "Degree-based topological indices," Croatica Chemica Acta, vol. 86, no. 4, pp. 351-361, 2013.

[15] M. Randic, "Characterization of molecular branching," Journal of the American Chemical Society, vol. 97, no. 23, pp. 6609-6615, 1975.

[16] M. Eliasi and B. Taeri, "Four new sums of graphs and their wiener indices," Discrete Applied Mathematics, vol. 157, no. 4, pp. 794-803, 2009.

[17] I. Gutman and N. Trinajstić, "Graph theory and molecular orbitals. Total $\varphi$-electron energy of alternant hydrocarbons," Chemical Physics Letters, vol. 17, no. 4, pp. 535-538, 1972.

[18] I. Gutman, B. Ruscic, N. Trinajstic, and C. F. Wilson, "Graph theory and molecular orbitals. XII. Acyclic polyenes," The Journal of Chemical Physics, vol. 62, no. 9, pp. 3399-3405, 1975.

[19] B. Borovicanin, K. C. Das, B. Furtula, and I. Gutman, "Bounds for Zagreb indices," MATCH Communications in Mathematical and in Computer Chemistry, vol. 158, pp. 1571-1578, 2010.

[20] K. C. Das and I. Gutman, "Some properties of the second Zagreb index," MATCH Communications in Mathematical and in Computer Chemistry, vol. 52, pp. 103-112, 2004.
[21] A. Ali and N. Trinajstic, "A novel/old modification of the first Zagreb index," Molecular Informatics, vol. 37, no. 6-7, Article ID $1800008,2018$.

[22] Z. Du, A. Ali, and N. Trinajstic, "Alkanes with the fist thee maximal/minimal modified fist zageb connection indices," Molecular Informatics, vol. 38, no. 4, Article ID 1800116, 2019.

[23] G. Ducoffe, R. Marinescu-Ghemeci, C. Obeja, A. Popa, and R. M. Tache, "Extremal graphs with respect to the modified first Zagreb connection index," in Proceedings of the 16th Cologne-Twente Workshop on Graphs and Combinatorial Optimization, pp. 65-68, CNAM, Paris, France, 2018.

[24] Z. Shao, I. Gutman, Z. Li, S. Wang, and P. Wu, Commun. Comb. Optim.vol. 3, pp. 179-194, 2018.

[25] J.-M. Zhu, N. Dehgardi, and X. Li, “The third leap Zagreb index for trees," Journal of Chemistry, vol. 2019, Article ID 9296401, 6 pages, 2019.

[26] J.-H. Tang, U. Ali, M. Javaid, and K. Shabbir, "Zagreb connection indices of subdivision and semi-total point operations on graphs," Journal of Chemistry, vol. 2019, Article ID 9846913, 14 pages, 2019.

[27] A. Graovac and T. Pisanski, "On the Wiener index of a graph," Journal of Mathematical Chemistry, vol. 8, no. 1, pp. 53-62, 1991.

[28] M. Azari and A. Iranmanesh, "Computing the eccentricdistance sum for graph operations," Discrete Applied Mathematics, vol. 161, no. 18, pp. 2827-2840, 2013.

[29] K. C. Das, A. Yurttas, M. Togan, A. S. Cevik, and I. N. Cangul, "The multiplicative Zagreb indices of graph operations," Journal of Inequalities and Applications, vol. 2013, no. 1, p. 90, 2013.

[30] N. De, A. Pal, and S. M. A. Nayeem, "On some bounds and exact formulae for connective eccentric indices of graphs under some graph operations," International Journal of Combinatorics, vol. 2014, Article ID 579257, 5 pages, 2014.

[31] M. H. Khalifeh, H. Yousefi-Azari, and A. R. Ashrafi, "The first and second Zagreb indices of some graph operations," Discrete Applied Mathematics, vol. 157, no. 4, pp. 804-811, 2009.

[32] J.-B. Liu, S. Javed, M. Javaid, and K. Shabbir, "Computing first general Zagreb index of operations on graphs," IEEE Access, vol. 7, pp. 47494-47502, 2019.

[33] J.-B. Liu, M. Javaid, and H. M. Awais, "Computing Zagreb indices of the subdivision-related generalized operations of graphs," IEEE Access, vol. 7, pp. 105479-105488, 2019.

[34] A. M. Naji and N. D. Soner, "The first leap Zagreb index of some graph operations," International Journal of Applied Graph Theory, vol. 2, pp. 07-18, 2018.

[35] M. Tavakoli, F. Rahbarnia, and A. R. Ashra, "Some new results on irregularity of graphs," Journal of Applied Mathematics \& Informatics, vol. 32, no. 5-6, pp. 675-685, 2014.

[36] M. Veylaki, M. J. Nikmehr, and H. A. Tavallaee, "The third and hyper-zagreb coindices of some graph operations," Journal of Applied Mathematics and Computing, vol. 50, no. 12, pp. 315-325, 2015.

[37] M. Ahmad, M. Saeed, M. Javaid, and M. Hussain, "Exact formula and improved bounds for general sum-connectivity index of graph-operations," IEEE Access, vol. 7, pp. 167290167299, 2019.

[38] X. Zhang, H. M. Awais, M. Javaid, and M. K. Siddiqui, "Multiplicative Zagreb indices of molecular graphs," Journal of Chemistry, vol. 201919 pages, 2019.

[39] A. R. Ashrafi, T. Došlić, and A. Hamzeh, "The Zagreb coindices of graph operations," Discrete Applied Mathematics, vol. 158, no. 15, pp. 1571-1578, 2010. 
[40] M. Dehmer, K. Varmuza, and D. Bonchev, Statistical Modeling of Molecular Descriptors in QSAR/QSPR, Wiley-VCH, Weinheim, Germany, 2012.

[41] M. V. Diudea, QSPR/QSAR Studies by Molecular Descriptors, NOVA, New York, NY, USA, 2001.

[42] J. Devillers and A. T. Balaban, Topological Indices and Related Descriptors in QSAR and QSPR, CRC Press, Amsterdam, Netherlands, 1999.

[43] I. Gutman and K. C. Das, "The first Zagreb index 30 years after," MATCH Communications in Mathematical and in Computer Chemistry, vol. 50, pp. 83-92, 2004.

[44] A. M. Naji, N. D. Soner, and I. Gutman, "On leap Zagreb indices of graphs," Communications in Combinatorics and Optimization, vol. 2, pp. 99-117, 2017.

[45] D. B. West, Introduction to Graph Theory, Prentice-Hall, Upper Saddle River, NJ, USA, 2nd edition, 2001.

[46] S. Yamaguchi, "Estimating the Zagreb indices and the spectral radius of triangle- and quadrangle-free connected graphs," Chemical Physics Letters, vol. 458, no. 4-6, pp. 396-398, 2008. 